\title{
Aeroacoustics of Darrieus wind turbine
}

\author{
Johannes Webera, ${ }^{*}$, Stefan Beckera, Christoph Scheita, \\ Jens Grabinger ${ }^{\mathrm{b}}$ and Manfred Kaltenbacher ${ }^{\mathrm{c}}$ \\ alnstitute of Process Machinery and Systems Engineering, Cauerstraße 4 , \\ 91058 Erlangen, Germany \\ ${ }^{\circ}$ Chair of Sensor Technology, Paul-Gordon-Straße 3/5, 91052 Erlangen, Germany \\ cInstitute of Mechanics and Mechatronics, Wiedner Hauptstraße 8, 1040 Vienna, Austria
}

Received: Jan. 20, 2014; Revised: Oct. 1, 2014; Accepted: Oct. 24, 2014

\begin{abstract}
The objective of this paper is to validate two different numerical methods for noise prediction of the H-Darrieus wind turbine using a complementary approach consisting of experimental measurements and numerical simulations. The acoustic measurements of a model scale rotor were performed in an anechoic wind tunnel. This data is the basis for the validation of the computational aeroacoustic simulations. Thereby, we have applied two different numerical schemes for noise prediction using hybrid methods. As usual in hybrid aeroacoustic approaches, flow field and acoustic calculations are carried out in separate software packages. For both schemes the time-dependent turbulent flow field is solved with Scale-Adaptive Simulation. The two schemes then differ in how the location of the acoustic sources and their propagation is calculated. In the first scheme the acoustic source terms are computed according to Lighthill's acoustic analogy which gives source terms located on the original CFD grid. These source terms are projected onto a coarser acoustic grid on which Lighthill's inhomogenous wave equation is solved by the Finite Element (FE) method. The second scheme uses the Ffowcs WilliamsHawkings (FW-H) method which is based on a free field Green's function. The scheme uses a porous integration surface and implements an advanced time formulation. Both methodologies are compared with experimental data.
\end{abstract}

\section{INTRODUCTION}

Wind energy is gaining importance in the last decades due to the emerging awareness of the need for environmentally sustainable power generation. The reason for this insight rests on the understanding of the finiteness of the fossil fuel reserves and of the negative effects of burning those fuels for energy production [1].

*wb@ipat.uni-erlangen.de 
Using onshore wind energy leads to the installation of more wind turbines in the vicinity of urban areas. Therefore, it is important to improve the performance with respect to sound emission in order to prevent noise pollution of residential areas. The term noise describes a subjective perception of sound waves by the human ear, which is unwanted. The hearing range of the average human ear is from $20 \mathrm{~Hz}$ to $20 \mathrm{kHz}$. Beside the conventional, large horizontal wind turbines, small wind turbines are considered as a possible solution for harvesting wind energy especially at small scales in urban areas. Investigations in this area so far have focused on vertical axis wind turbines (VAWT), which in comparison to horizontal axis wind turbines are characterized by the following advantages [2]:

- Insensitivity to wind direction, which avoids the need of a yaw system

- Applicability in presence of turbulent streams

- Lower manufacturing costs of VAWTs [3]

The most widely known design of VAWTs is the Darrieus turbine, which was developed by the French engineer Darrieus in the early $20^{\text {th }}$ century [2]. This type is driven by lift force, which is the most efficient way to convert wind energy into mechanical energy. Contrary to that, a typical drag-type device is the Savonius rotor. Due to the low power coefficient of maximally $30 \%$, the Savonius rotor doesn't apply to commercial wind energy use [2].

\section{WIND TURBINE NOISE}

Due to the increasing power and the increasing noise levels of wind turbines during the last decades, investigating the noise mechanisms of wind turbines is still an important area of research. In general, there are different sound sources of wind turbines. Those can be divided into two groups. The first noise source to mention is the mechanical noise, which is produced by e.g. the gearbox, generator, yaw drives, cooling fans and auxiliarly equipment like hydraulics. The acoustic transmission pathway of mechanical noise can be a type of air-borne or structure-borne sound. The second sound source, which is object of this investigation, is the aerodynamic noise. Its character is on the one hand of tonal and on the other hand of broadband nature. Both depend strongly on the geometry of the rotor, the shape of the airfoils and their surrounding flow conditions. The aerodynamic sound sources are typically produced by the flow of air over the blades. It can be separated into three categories: Turbulent inflow noise, Airfoil self-noise and low frequency noise. Turbulent inflow noise occurs due to the atmospheric turbulence in form of local pressure fluctuations interact with the blades and contributes mainly to broadband noise. Airfoil self-noise is generated by the interaction between the airfoil and the turbulence induced in its own boundary layer and near wake. Different airfoil self-noise mechanisms, which show either tonal or broadband sound characteristics, are presented in the following:

- Turbulent-boundary-layer - trailing edge noise

- Laminar-boundary-layer - vortex shedding noise

- Separation stall noise

- Trailing-edge-bluntness - vortex shedding noise

- Tip vortex formation noise 
Further information of each mechanism will be given in the literature by Brooks, Pope and Marcolini [4]. Low frequency noise is considered as sound, which is generated when the rotating blades interfere with localized flow changes due to the tower, wind speed variations or wakes, which are caused by other blades. The characteristic frequency range of low frequency noise is from about $10 \mathrm{~Hz}$ to $200 \mathrm{~Hz}$. Low frequency noise is relating mainly to the blade passing frequency and its higher harmonics [5].

\section{STATE OF THE ART OF VAWTS AND PURPOSE OF THIS WORK}

In recent years, experimental and numerical investigations, which are mostly focused on the aerodynamic behaviour, were performed in the field of VAWTs, e.g., by Mohamed, who investigated the aerodynamic performance of the H-Darrieus turbine with different airfoil shapes [6]. Simão Ferreira placed the focus on the research of dynamic stall [7]. Mertens investigated the performance of an H-Darrieus in the skewed flow on a roof [8]. Marnett studied a multiobjective numerical design of vertical axis wind turbine components in his $\mathrm{PhD}$ thesis [9]. Beside of the aerodynamic investigations of VAWTs, just a few publications on noise emission are available today. Iida investigated numerically the aerodynamic sound of a VAWT by using discrete vortex methods. This paper pointed out that a HAWT generates more noise as a VAWT with the same power coefficient at normal operating speed [10]. In 2013, Pearson has performed an investigation of the noise sources on a VAWT using an acoustic array. He stated that at low frequencies the harmonic components are dominating the spectrum. At low tip-speed ratio the harmonics were much stronger in comparison to those at higher tip-speed ratio. Therefore, Pearson suggested that at low tip-speed ratio the unsteady blade loading is much higher due to the dynamic stall effect [11]. Mohamed investigated numerically the noise emission of a Darrieus turbine using a FfowcsWilliams and Hawkings method. This paper shows a parameter study, in which the blade shape, tip-speed ratio and solidity effects were varied. He came to the conclusion, that the higher tip-speed ratio and higher solidity rotors generate more noise than normal turbines [12].

In order to get valid numerical approaches to predict the aeroacoustics of the H-Darrieus turbine, a complementary approach consisting of experimental measurements, computational fluid dynamics simulations and aeroacoustics simulations were performed in this work. After measuring experimental data, a transient flow simulation was carried out in the commercial computational fluid dynamics (CFD) software ANSYS-CFX [13]. Following that, two different in-house codes were used for acoustic post-processing. The first code is the finite-element multiphysics solver CFS++ [14], which is based on Lighthill's analogy. The second one is called SPySI (sound prediction by surface integration), which is predicated on the porous Ffowcs Williams-Hawkings method [15]. Such tools for noise prediction can be very useful in wind turbine design in order to optimize the system with respect to noise and aerodynamic performance. The reason of this work is the validation of two different inhouse-codes used for noise prediction in a first step. Therefore the focus is placed mainly on the aeroacoustics of the H-Darrieus turbine. 


\section{EXPERIMENTAL SETUP}

The experimental measurements took place in an anechoic wind tunnel at the University of Erlangen-Nürnberg, which is schematically depicted in figure 1. The aeroacoustic wind tunnel is characterized of the closed return with an open test section.

(a)

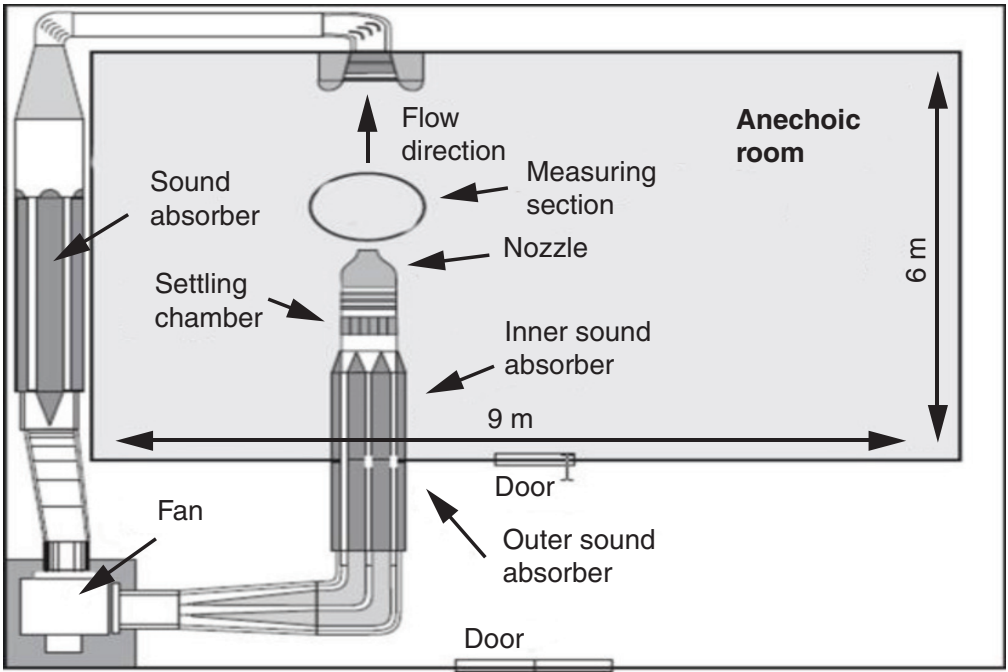

(b)

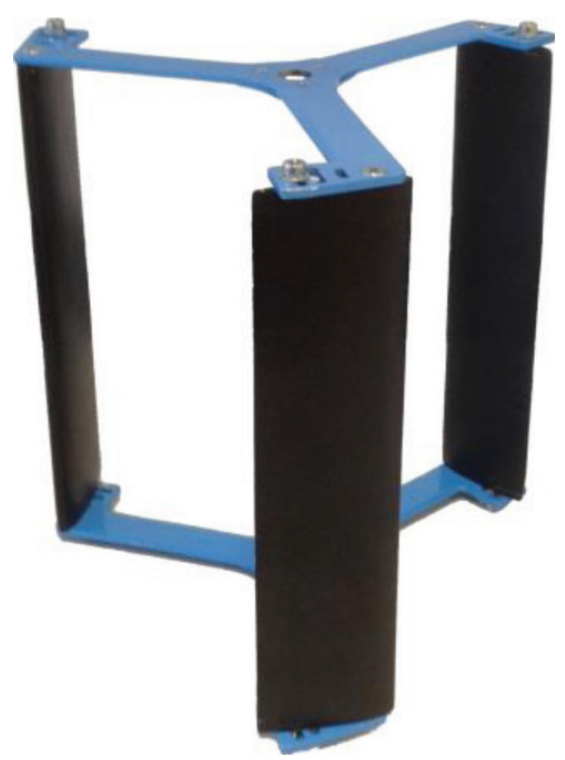

Figure 1: $\quad$ Anechoic wind tunnel (a) and the model scale rotor (b). 
This section is located in an anechoic chamber, so that free field acoustic measurements without any reflections from the walls can be performed. The absorption coefficient of this chamber has a factor of 0.9 for a frequency of $300 \mathrm{~Hz}$. In order to assure a low noise level in the test section, the wind tunnel possess silencers to damp out fan noise. The nozzle of the wind tunnel has a cross-section area of $0.25 \mathrm{~m} \times 0.33 \mathrm{~m}$ and achieves a maximum wind speed of $35 \mathrm{~m} / \mathrm{s}$. A low turbulence level of $0.15 \%$ in the wind tunnel is accomplished because of several turbulence grids and a honeycomb. The subject of these investigations is a generic model scale rotor of a 3-bladed H-Darrieus turbine as illustrated in fig. 1. The airfoil profile NACA0018 was chosen because of its good aerodynamic performance for VAWTs as described in literature [16]. Due to the small wind tunnel working section, a model of $0.2 \mathrm{~m}$ diameter and height is used. The chord length $\mathrm{c}$ of the model was chosen as $0.05 \mathrm{~m}$. In order to issue physically valid statements about larger rotors, Reynolds number similarity has to be fulfilled. The maximum achievable Reynolds number referenced to the diameter $\mathrm{d}\left(R e_{d}=\rho v d / \eta\right)$ calculated with the maximum wind speed of $35 \mathrm{~m} / \mathrm{s}$ of the wind tunnel leads to 466000 . Assuming a real rotor configuration of $1 \mathrm{~m}$ height and diameter this Reynolds number would be achieved at a wind speed of $v=7 \mathrm{~m} / \mathrm{s}$.

For example such small rotors could be roof mounted at single-family houses for producing energy. Further rotor details of the full scale rotor and the model are illustrated in table 1 . In order to characterize the blade aerodynamics and the relevant noise mechanisms the Reynolds number will be now referred to chord length $\mathrm{c}$ and the angular velocity $\omega=2 \pi n r$. In case of the validation test case $(v=21,28 \mathrm{~m} / \mathrm{s})$, which we present in the following sections, the Reynolds number reaches a value of $R e_{c}=28000$ at a rotating speed of $800 \mathrm{rpm}$. This Reynolds number is very low considering the typically range between $0.25 \times 10^{6}$ and $1.0 \times 10^{6}[16]$ and therefore strong dynamic stall effects will be expected for the investigated operating condition. Furthermore, no turbulence generators were used to force transition at a certain position of the airfoil. But as we mentioned in the introduction section, this study focus on the validation of the acoustic simulation results and shall only explain the fundamental acoustic mechanisms at this operating point. Anyway, we believe that also in this test case the basic mechanisms of the acoustics can be explained if one compares the sound pressure spectra with the results of Pearson [11].

One of the most important quantities for the aerodynamic performance is the rotor solidity, which is defined as $\sigma=N c /(2 \mathrm{r})$ where $\mathrm{N}$ represents the number of blades, c the chord length of the blade and $\mathrm{r}$ is the radius. It describes how much of the horizontal turbine projection area $A=2 h r$ is covered by the airfoils which in turn affects the amount of deflection of the incoming flow [9].

Table 1: Comparison of the geometric quantities between full rotor design and $1 / 5^{\text {th }}$ model scale rotor.

\begin{tabular}{lccccc} 
Rotor & $\mathbf{h}[\mathbf{m}]$ & $\mathbf{d ~}[\mathbf{m}]$ & $\mathbf{v}[\mathbf{m} / \mathbf{s}]$ & $\mathbf{R e}_{\mathbf{d}}[-]$ & Solidity $\boldsymbol{\sigma}[-]$ \\
\hline Full-scale & 1 & 1 & 7 & 466000 & 0.75 \\
Model & 0.2 & 0.2 & 35 & 466000 & 0.75
\end{tabular}


In order to measure the acoustic pressure, four 1/2-inch free-field microphones (Bruel \& Kjaer type 4189) were positioned in a half circle in equal angles of 45 degrees at a distance of $1=1 \mathrm{~m}$ as depicted in fig. 2 . The microphones have a linear frequency response characteristic. Furthermore, the frequency spectrum ranges between $6.3 \mathrm{~Hz}$ and

(a)

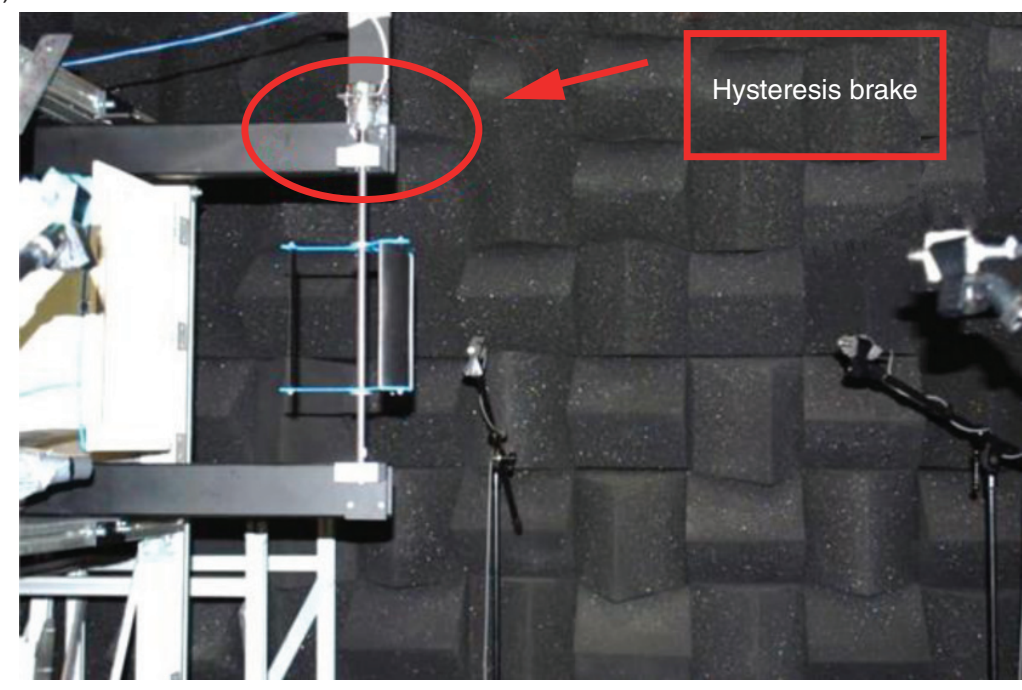

(b)

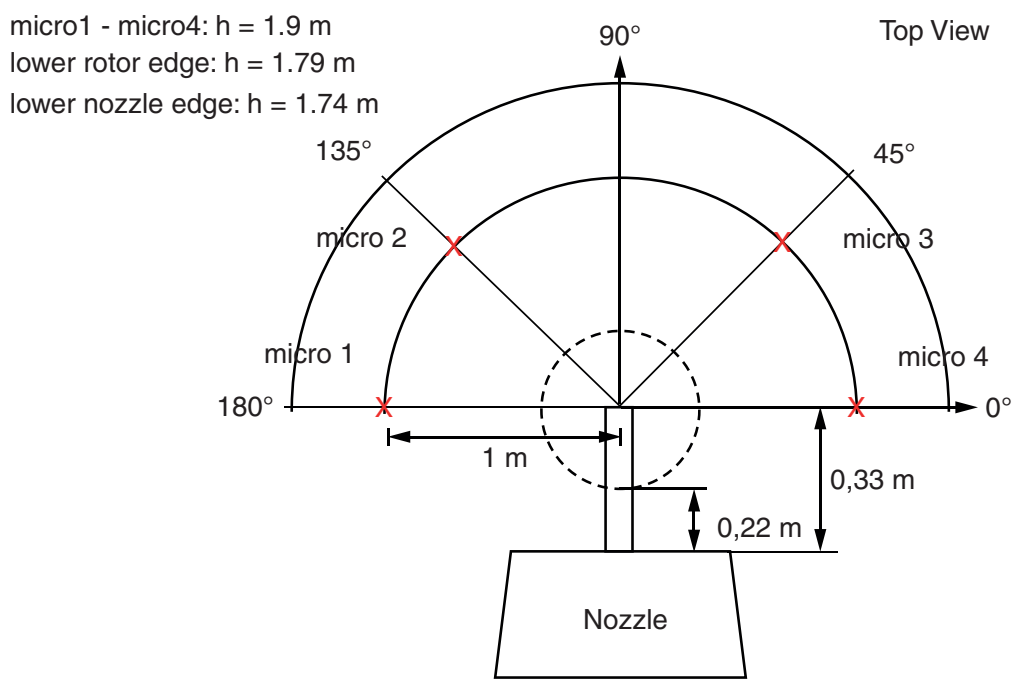

Figure 2: Experimental set-up (a) and a schematically drawing of the set-up in top view (b). 
$20 \mathrm{kHz}$ and the dynamic range is from $14.6 \mathrm{~dB}$ to $146 \mathrm{~dB}$. These were connected with a Nexus amplifier type 2690-A-0S4 of Bruel \& Kjaer. In order to convert the signal, a National Instrument PXIe-4492 A/D converter was used. The height of the microphones was chosen as $\mathrm{h}=1.9 \mathrm{~m}$, which is the middle of the rotor height. The distance between the outlet of the nozzle and the center of the model is $\mathrm{s}=0.33 \mathrm{~m}$. The torque of the model scale rotor was measured with a hysteresis brake. By measuring the pressure drop along the nozzle and applying Bernoulli's formula the desired wind speed is adjusted.

\section{METHODS}

\subsection{Numerical methods}

\subsubsection{Aerodynamic simulation}

The experimental measurements are validated with the help of the CFD simulations. Therefore, the design of an H-Darrieus wind turbine as illustrated in fig. 2 is used for the turbulent flow field computation by the finite-volume method solver ANSYS-CFX 14.0. The wind turbine consists of three symmetric NACA0018 airfoils, which are uniformly distributed in circumferential direction. Figure 3 shows the circumferential computational
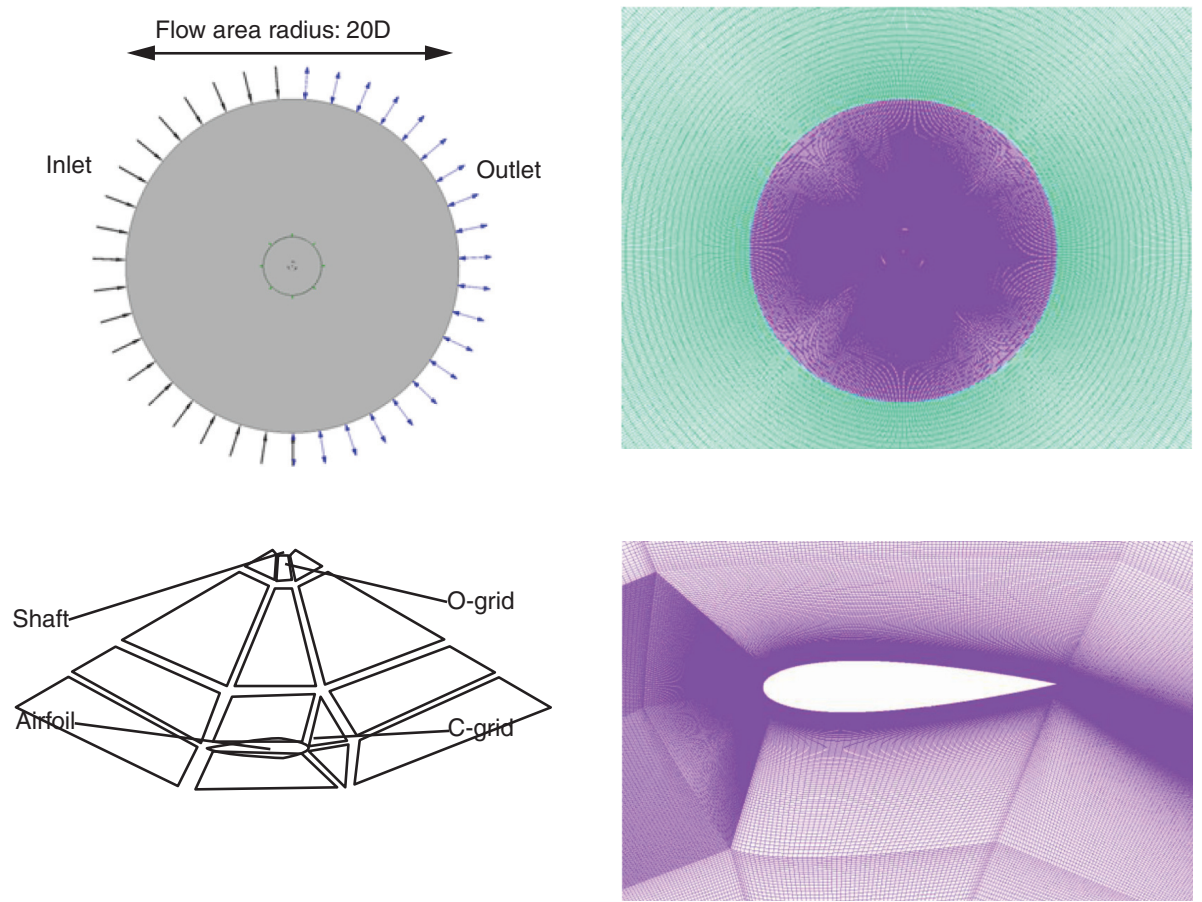

Figure 3: Computational domain of the CFD-simulation (top left) and illustration of the block strategy (bottom left), the mesh topology at the rotating and stationary region (top right) and the close-up view of the airfoil (bottom right). 
fluid domain composed of a rotating and a stationary region, which are connected by a transient rotor stator interface. The inlet of the fluid domain is located on the left half of the outer circle and the inlet boundary condition was given by $\mathrm{v}=21.28 \mathrm{~m} / \mathrm{s}$ wind speed. At the outlet, an opening boundary condition was defined at relative pressure $0 \mathrm{~Pa}$ in order to allow backflow that means vortices are permitted to pass the outlet boundary. This results in in- and outflow at the same time. The rotational speed of the airfoils was set to $n=800 \mathrm{rpm}$. At this operating point a tip-speed ratio $\lambda=2 \pi n r / v$ of 0.4 is achieved. That means that high dynamic stall occurs at the airfoils [2].

A hexahedral mesh consisting of 1.4 Mio cells, of which 1.3 Mio cells are located in the rotating region, was generated in ANSYS ICEM. Due to the rotational symmetry of the rotor, one third of the turbine was meshed and rotated to the full 360 degree geometry. Conforming, periodic interfaces were placed at the outer edges of the grid in order to make sure that every node matches after the rotation of the mesh. Ensuring that the boundary layers on the blades and on the shaft are adequately resolved, the mesh is strongly refined to obtain a normalized distance of the wall nearest grid cell of $y^{+}<1$. Therefore, the first cell size around the blade was chosen about $2 \mu \mathrm{m}$ in surface normal direction and 30 layers are positioned in the boundary layer. A close-up view of the airfoil is depicted in fig. 3. In order to affirm mesh independency, a grid study was performed prior to this work.

Because the simplified Lighthill stress tensor formulation used in CFS++ assumes a constant density while the Ffowcs Williams-Hawkings method of SPiSY requires a variable one, both an incompressible and a compressible simulation were run.

Both simulations are solved with Scale-Adaptive Simulation (SAS) established by Menter and Egorov [17, 18]. The reason for choosing the SAS model was to assure that also smaller structures can be resolved, which may have an influence on the acoustics. The SAS-turbulence-model is an extension to the unsteady RANS model, which is also able to resolve turbulent structures in a Large Eddy Simulation (LES)-like behavior, while URANS methods only resolve unsteady, mean flow structures like coarse vortices. The URANS approach is based on the separation of the flow field quantities in time averaged quantities and fluctuations. The SAS model uses a blending from this RANS approach to a scale-resolving approach. This blending is a function of the cell size. The coarser the grid size is, the bigger the influence of the RANS approach is, while fine grid sizes lead to resolution of small turbulent structures. As spatial discretization scheme the bounded central difference scheme is used. A time step of $\Delta t=1 e^{-5} s$ was chosen, which corresponds to $C F L \sim 1$ [19] and to an azimuth angle of 0.048 degrees. A second order backward Euler scheme was applied for the temporal discretization. In order to initialize the SAS simulation it is recommended by Menter using a RANS model solution [28]. Therefore, the result of a previous URANS simulation was used as initial condition. Both simulations offer the same grid and boundary conditions except for the time step, which was $\Delta t=1 e^{-4} s$ in case of the URANS. As convergence criterion the RMS value of the momentum and mass residuals were chosen. Five inner iterations were performed within each time step to decrease these residuals. Stable flow conditions were obtained after three revolutions. Six pressure and velocity in stationary frame probes located at the airfoils were monitored. 
At each monitor point a periodic signal could be observed. The physical simulation time of both simulations was $1.2 \mathrm{~s}$. After ensuring that the flow field was fully developed, 30000 time steps were calculated for the acoustic simulation. At every tenth of these flow field data was exported, which amounts to 3000 time steps of $1 e^{-4} s$ for the acoustic simulations. Because of placing the main focus of this work on the validation of the acoustic simulations, no aerodynamic properties like lift coefficient or drag coefficient was considered.

\subsubsection{Aeroacoustic formulation}

The age of modern aeroacoustics is considered to begin with the seminal work of Lighthill in 1952 [20] and 1954 [21] on sound generated aerodynamically by jet engines. In his two-part paper he derived the concept of the acoustic analogy, resulting in the inhomogeneous wave equation, which has been solved by an integral representation using a free-space Green's function. Subsequently, Curle (1955) extended Lighthill's integral representation to take into account the influence of solid bodies [22]. In many technical applications such as helicopter rotors, aeroplane propellers, fans and turbines, moving solid surfaces are directly involved in the generation of noise. This is considered by the extension of Ffowcs Williams and Hawkings (1969) [23].

In order to calculate the sound pressure spectra radiated by the H-Darrieus, the acoustic analogies established by Lighthill on one hand and Ffowcs Williams and Hawkings on the other hand are used and will be presented in the following.

The computation of flow-induced noise according to Lighhill's analogy starts with his famous inhomogeneous wave equation for the sound pressure $p^{\prime}$

$$
\frac{1}{c_{0}^{2}} \frac{\partial^{2} p^{\prime}}{\partial t^{2}}-\frac{\partial p^{\prime}}{\partial x_{i}^{2}}=\frac{\partial^{2} T_{i j}}{\partial x_{i} \partial x_{j}}
$$

The left hand side is equivalent to the homogenous wave equation. The right hand side which is the Lighthill tensor $T_{i j}$ is the source term of the complete inhomogeneous wave equation. It was derived by Lighthill directly from the conservation of mass and momentum.

$$
T_{i j}=P_{i j}+\rho u_{i} u_{j}-c_{0}^{2} \delta_{i j} \rho^{\prime}=\rho u_{i} u_{j}+\left(p^{\prime}-c_{0}^{2} \rho^{\prime}\right) \delta_{i j}-\tau_{i j},
$$

which consists of non-linear convective forces $\rho u_{i} u_{j}$, deviations in the speed of sound $c_{0},\left(p^{\prime}-c_{0}^{2} \rho^{\prime}\right)$, viscous forces $\tau_{i j}$ and the Kronecker delta $\delta_{i j}$.

A further simplification of the Lighthill tensor can be accomplished in case of an isotropic flow at low Mach numbers. In this case, viscous effects $\tau_{i j}$ are negligible. Also, the term $\left(p^{\prime}-c_{0}^{2} \rho^{\prime}\right)$ is only relevant for anisotropic media and can be considered to be very small in air. Only the non-linear convective effects $\rho u_{i} u_{j}$ remain as sound sources which results in

$$
T_{i j} \approx \rho_{0} u_{i} u_{j}
$$


Since we solve directly the Lighthill's inhomgeneous wave equation by the FE method, we implicitely take all acoustic source mechanism into account. To obtain a formulation suitable for finite element-methods, a weak formulation of Lighthill's inhomogeneous wave equation is developed. For this purpose, eq. (1) is multiplied by an appropriate element shape function w, integrated over the computational domain $\Omega$ and Stokes' integral theorem is applied.

$$
\int_{\Omega} \frac{1}{c_{0}^{2}} \frac{\partial^{2} p^{\prime}}{\delta t^{2}} \mathrm{w} \mathrm{d} \Omega+\int_{\Omega} \frac{\partial w}{\partial x_{i}} \frac{\partial p^{\prime}}{\partial x_{i}} \mathrm{~d} \Omega-\int_{\Gamma} \frac{\partial p^{\prime}}{\partial n} w \mathrm{~d} \Gamma=-\int_{\Omega} \frac{\partial w}{\partial x_{i}} \frac{\partial T_{i j}}{\partial x_{j}} \mathrm{~d} \Omega
$$

For details concerning the FE formulation, we refer to [14].

As a second approach, we have chosen the porous Ffowcs Williams-Hawkings method, which is based on an integral solution of eq. (1). In comparison to the formulation of Lighthills equation not only the transient flow velocity, but also the pressure and density data is needed. Furthermore, a surface integral $S$ wrapped around the source region $V$, which is defined by the scalar function $f\left(x_{i}, t\right)$ (see fig. 4 ),

$$
\begin{aligned}
& f\left(x_{i}, t\right)<0 \quad \text { if } x_{i} \text { is placed inside of the source region } V \\
& f\left(x_{i}, t\right)>0 \quad \text { if } x_{i} \text { is placed outside of the source region } V \\
& f\left(x_{i}, t\right)=0 \quad \text { if } x_{i} \text { is placed on the source region } S
\end{aligned}
$$

has to be chosen. The Heaviside function $H(f), H(f)=1$ for $f>0, H(f)=0$ for $f<0$, ensures that no boundary conditions have to be fulfilled on the boundaries.

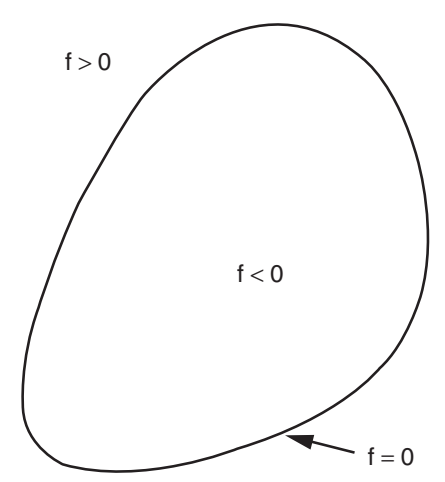

Figure 4: Boundary of a solid body inside the flow domain described by scalar function $\mathrm{f}[15]$. 
If one multiplies the Heaviside function $H(f)$ with the basic equations in the derivation of Lighthill's analogy, one obtains

$$
\begin{aligned}
& \frac{\partial^{2}\left\{\left(\rho^{\prime}\right) H(f)\right\}}{\partial t^{2}}-c_{0}^{2} \frac{\partial^{2}}{\partial x_{i} x_{j}}\left\{\left(\rho^{\prime}\right) \delta_{i j} H(f)\right\} \\
& =\frac{\partial^{2}}{\partial x_{i} x_{j}}\left\{T_{i j} H(f)\right\}+\frac{\partial}{\partial t}\left\{\left(\rho\left(u_{i}-v_{i}^{S}\right)+\rho_{0} v_{i}^{S}\right) \delta(f) \frac{\partial f}{\partial x_{i}}\right\} \\
& -\frac{\partial}{\partial x_{i}}\left\{\left(P_{i j}+\rho u_{j}\left(u_{i}-v_{i}^{S}\right)\right) \delta(f) \frac{\partial f}{\partial x_{i}}\right\}
\end{aligned}
$$

Equation (6) is known as the differential form of Ffowcs Williams-Hawkings, which expresses a generalized form of the Lighthill equation. Here, $P_{i j}$ represents the compressible stress tensor, $u_{i}$ corresponds to the flow velocity, $v_{i}^{s}$ symbolizes the integration surface velocity and $\delta(f)$ is the Dirac function, which is the derivative of the Heaviside function. For calculation of radiation into free field eq. (6) can be solved with the free-space Green's function [24],

$$
G(x, t)=\frac{\delta\left(t-\left|x_{i}-y_{i}\right|-\tau / c_{0}\right)}{4 \pi\left|x_{i}-y_{i}\right|},
$$

where $c_{0}$ represents the ambient speed of sound and $\tau$ is the retarded time. Inside SPySI, Farassat's formulation 1 [25] is implemented. For a stationary, porous integration surface $\mathrm{S}$, the solution can be written as

$$
p^{\prime}\left(x_{i}, t\right)=p_{Q}^{\prime}\left(x_{i}, t\right)+p_{L}^{\prime}\left(x_{i}, t\right)+p_{T}^{\prime}\left(x_{i}, t\right)
$$

Here $p_{T}^{\prime}$ denotes the thickness noise and represents the physical mechanism of displacement of the fluid in the flow field by solid surfaces such as turbine blades. The term loading noise $p_{L}^{\prime}$ describes the physical mechanism of the force that acts on the fluid as a result of the presence of the surfaces of the body. The quadrupole distribution is denoted as $p_{Q}^{\prime}$.

If volume forces are neglected and an integration over the surface $\mathrm{S}$ is performed, solutions for the thickness noise and loading noise are obtained:

$$
\begin{gathered}
4 \pi p_{T}^{\prime}\left(x_{i}, t\right)=\frac{\partial}{\partial t} \int_{S}\left[\frac{\rho_{0} U_{n}}{r}\right]_{r e t} d S \\
4 \pi p_{L}^{\prime}\left(x_{i}, t\right)=\frac{1}{c_{0}} \frac{\partial}{\partial t} \int_{S}\left[\frac{L_{r}}{r}\right]_{r e t} d S+\int_{S}\left[\frac{L_{r}}{r^{2}}\right]_{r e t} d S
\end{gathered}
$$


The distance between the surface source and the observer position is represented by $r$ and subscript $n$ defines scalar contraction with the integration surface normal vector $n_{i}$. The variables $U_{i}$ and $L_{i}$ are introduced by Di Francescantonio [26] as following:

$$
U_{i}=\frac{\rho}{\rho_{0}} u_{i} ; \quad L_{i}=P_{i j} n_{j}+\rho u_{i} u_{n}
$$

To evaluate the wave propagation time between source and observer, there are two possible algorithms: the retarded time approach and the advanced time approach. In case of the advanced time algorithm

$$
t_{a d v}=t+\frac{r(t)}{c_{0}}
$$

only one time step of CFD simulation data per acoustic evaluation is needed. Contrary, the retarded time algorithm requires several time steps at different source positions and therefore multiple time steps have to be stored in memory simultaneously. SPySI uses the advanced time algorithm.

\section{RESULTS AND DISCUSSION}

\subsection{Experimental results}

In fig. 5 the measured sound pressure level spectrum at microphone 1 is illustrated. The operating point of this measurement is at a Reynolds number of 28000 at wind speed of $21.28 \mathrm{~m} / \mathrm{s}$. A major peak can be seen at the blade passing frequency (BPF) of $40 \mathrm{~Hz}$.

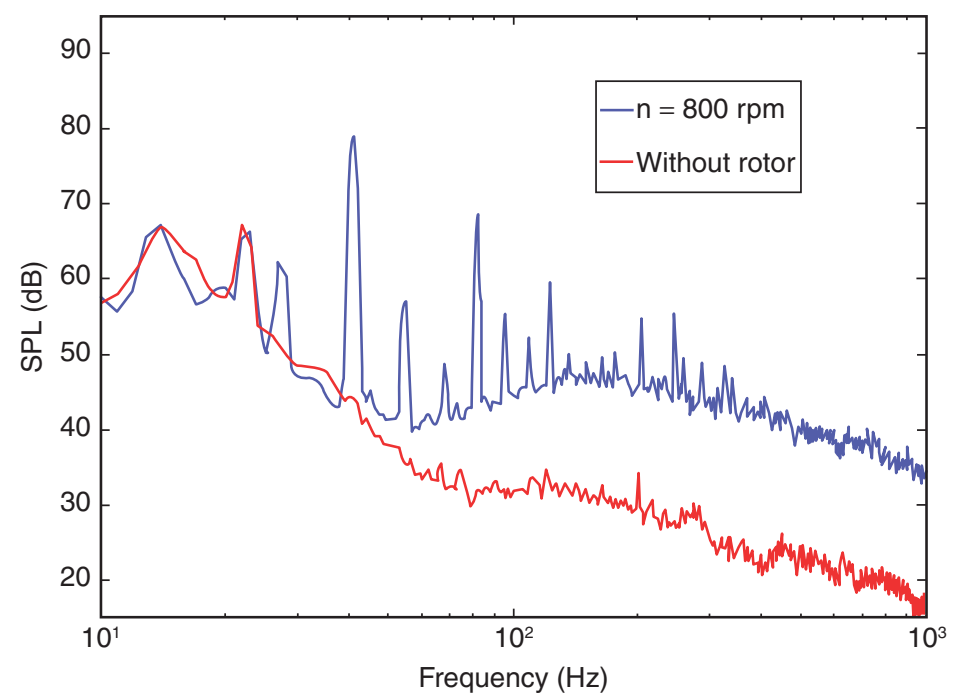

Figure 5: Experimental data at rotating speed of $800 \mathrm{rpm}$ (blue) and the reference measurement without any rotor. 
Further peaks of the harmonics of the BPF are visible at $80 \mathrm{~Hz}$ and $120 \mathrm{~Hz}$. Between these harmonics small peaks can be seen which refer to the noise of the bearings. Furthermore, broadband noise appears at around $200 \mathrm{~Hz}$ and becomes dominant at frequencies above $400 \mathrm{~Hz}$. A reference measurement has been carried out in order to distinguish if sound spectrum components are either caused by the wind tunnel or the Darrieus rotor. The reference measurement contains only the noise caused by the wind tunnel. The comparison to the full noise spectrum shows that at frequencies smaller than $30 \mathrm{~Hz}$ the wind tunnel noise is dominant.

\subsection{Turbulent flow field and acoustic sources}

To illustrate the transient flow field obtained by the CFD simulation, the evolution of the flow is presented in fig. 6 . The left series shows the temporal evolution of the velocity in the stationary frame, the right series depicts the pressure (the flow direction of the air is from the left side to the right side in the pictures).

While the airfoils are moving upstream, (airfoil top left, 40 and 80 degrees) highly turbulent structures can be seen in the flow as well as in the pressure field downstream and at the inner region of the Darrieus. A major part of this turbulence is induced by stall. After passing the most upstream (airfoil left, 80 degrees) or the most downstream
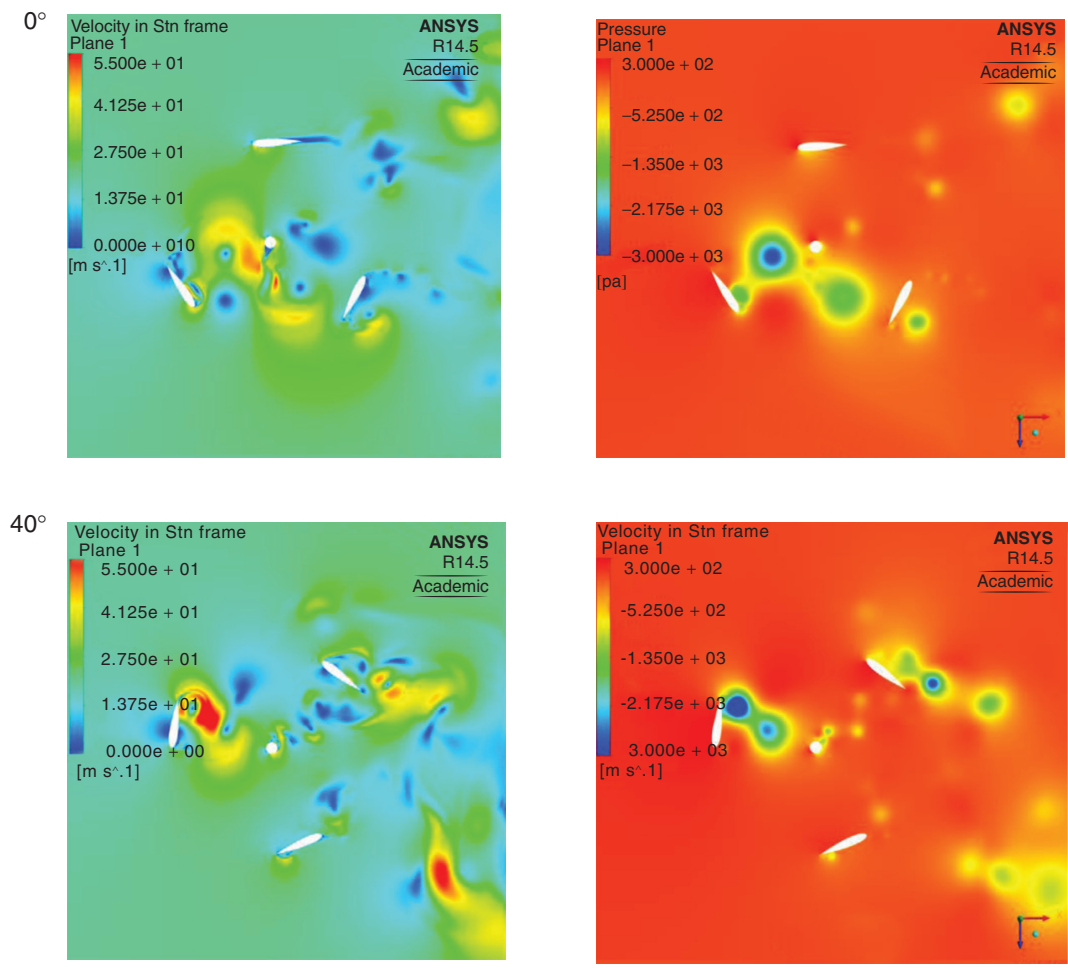

Figure 6(continued) 
$80^{\circ}$

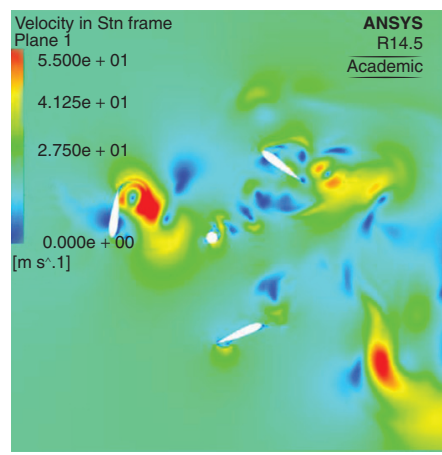

$120^{\circ}$

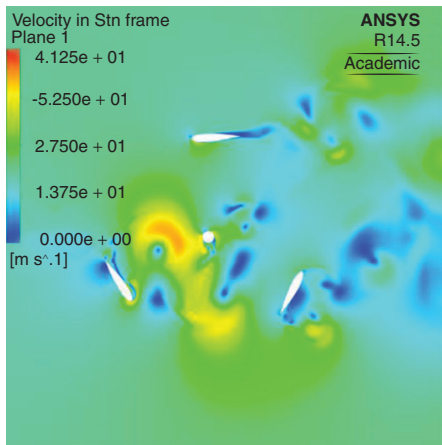

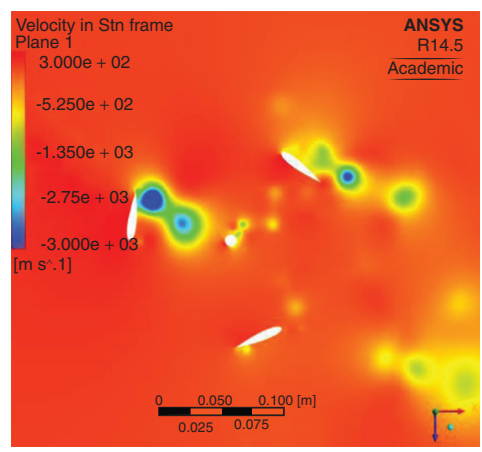

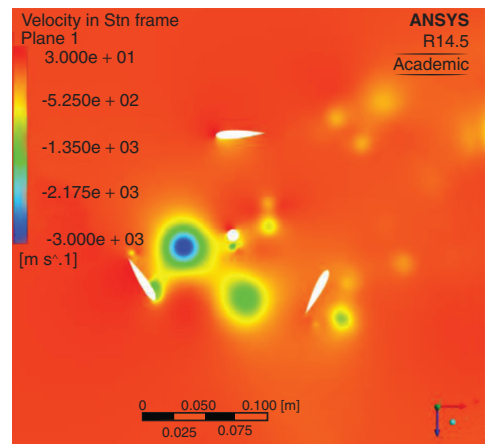

Figure 6: Temporal development of the velocity in stationary frame (left) and pressure (right) in the rotating domain.

(airfoil right, 40 degrees) position, large vortices separate from the airfoils due to the stall effect. The vortices induced upstream hit the shaft and decompose into smaller vortices. Beside of this, the following blades interfere with the wakes from the upstream blades. This induces impulsive blade loads, which are called blade-vortex interaction. Furthermore, the shaft itself is a source of vortex generation and separation.

In fig. 7 the temporal development of the dimensionless pressure distribution at one blade is shown, which is defined as $p^{*}=p / 0.5 \rho v^{2}$. At the azimuth angle of $0^{\circ}$ the blade experiences almost no big pressure differences as usual for symmetric airfoils. If the blade rotates to an angle of $40^{\circ}$ the pressure of the lower surface exhibits a vortex in the near of the leading edge. At an angle of $80^{\circ}$ this vortex floating downstream to the trailing edge. In case of $120^{\circ}$ a new vortex formation grows at the leading edge. 

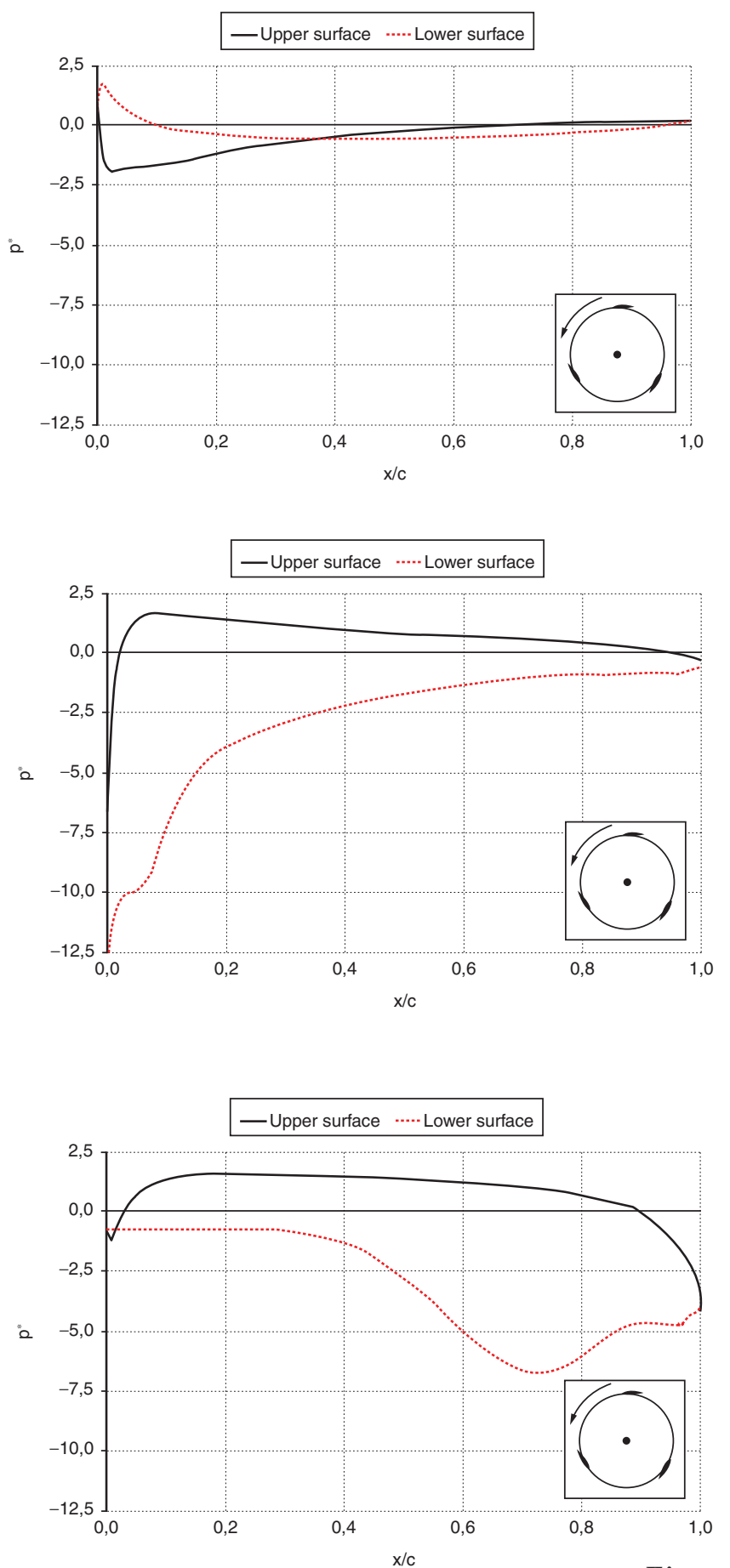

Figure 7(continued) 


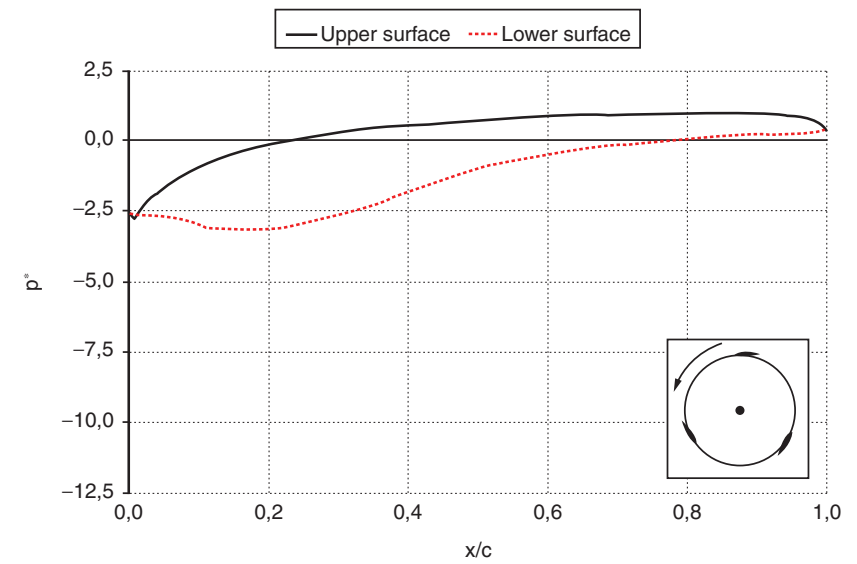

Figure 7: Temporal development of the pressure distribution at different azimuth angles.

In fig. 8, the temporal evolution of the acoustic source terms, which are computed on the original CFD grid, is depicted. These images show the influence of the previously described flow phenomena on the acoustics.

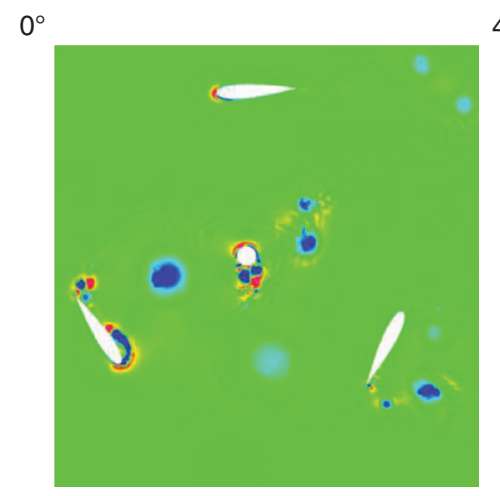

$40^{\circ}$
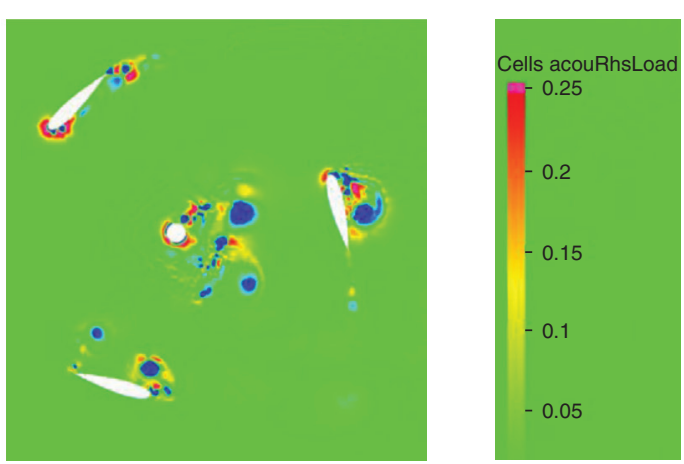

$80^{\circ}$

$120^{\circ}$
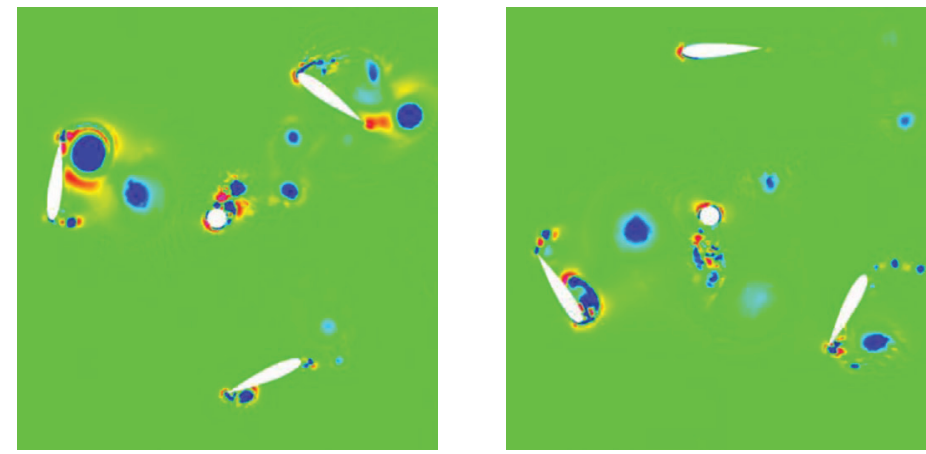

Figure 8: Temporal development of the acoustic source terms. 
The Karman vortices appearing at the shaft appeared to be a further sound source. Vortex separation at the inner side (airfoil left, 80 degrees) of the blade and the outer side (airfoil right, 40 degrees) are another important sound source [4].

\subsection{Validation with experiments}

A single FFT was applied to both numerical results, in order to get a spectral comparison between the FE simulation on one hand and the FW-H approach on the other hand, which one can see in figure 9 and 10. While the overall sound pressure level for frequencies higher than $100 \mathrm{~Hz}$ is overpredicted by the FWH approach, the FE simulation underestimates the sound pressure level in this frequency range. The FE-Simulation captures the height of the amplitude of the BPF at $40 \mathrm{~Hz}$ in good agreement, but the peak is not as discrete as in the case of FWH. The first harmonic at $80 \mathrm{~Hz}$ is captured as well. The results of FWH show, that the BPF and the first harmonic are also resolved, but of lower amplitude. The overall sound spectrum level shows a discrepancy of $9 \%$ in case of the FE-Simulation and 7\% in case of FWH. In general, the noise mechanisms at this low tip-speed ratio can be referred to the blade vortex interaction noise, which is resulting in the blade passing frequency and its higher harmonics. "Thickness noise" corresponds to the blade passing frequency, which describes the displacement of fluid by the blade. Due to the higher harmonics, it is expected that "loading noise" has an impact on the general noise, which is caused by different lift and drag forces on the rotating blade. At lower tip-speed ratio the airfoils perceive severe dynamic stall, which means large and strong vortices are shed. The following, downstream blade interacts with the vortices of the previous airfoil and experiences massive force changes, which results in the generation of blade-vortex interaction noise. Beside of this, large flow separations take place at the blades of the

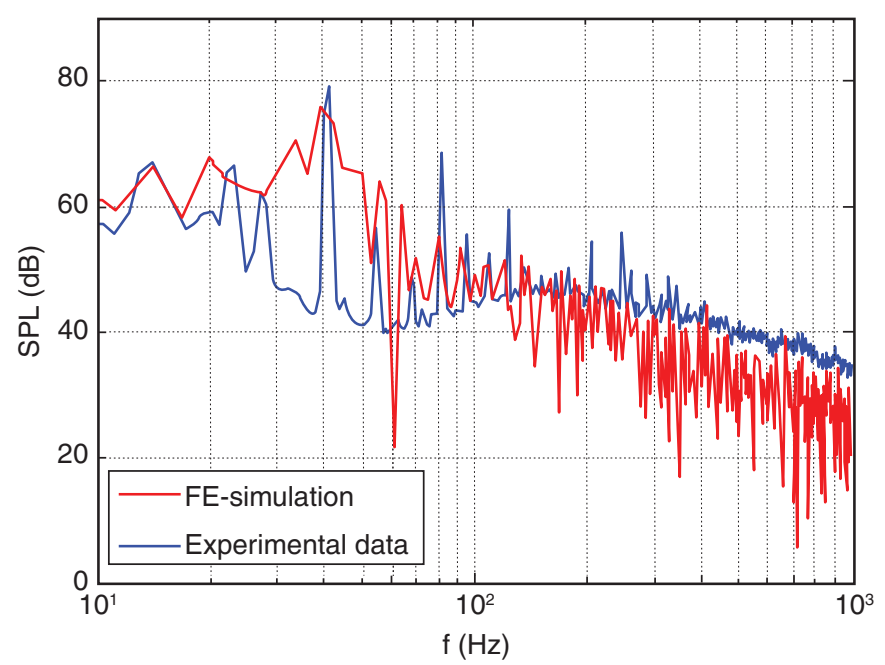

Figure 9: Sound pressure level spectra of $\mathrm{CFS}++$ and experimental data. 


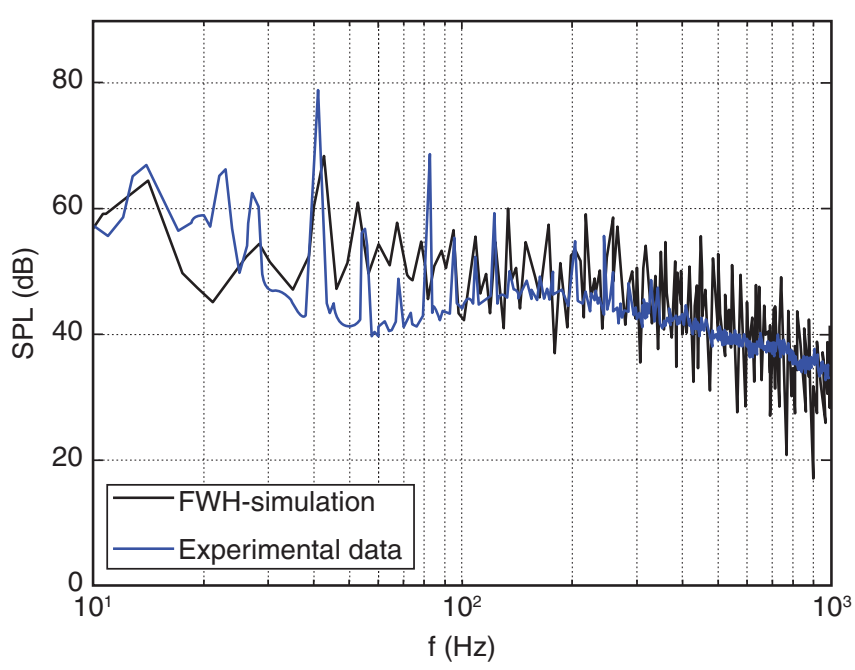

Figure 10: Sound pressure level spectra of FWH and experimental data.

darrieus turbine even at small angles of attack due to the small Reynolds number of this operating point and therefore separation stall noise will also have an impact on the total noise emission.

\section{CONCLUSION}

Numerical and experimental investigations of sound generation of an H-Darrieus wind turbine have been performed in order to validate the two different inhouse-codes. To this end, acoustic analogies of Lighthill and Ffowcs Williams and Hawkings have been applied to CFD simulation data. In summary, there is a good agreement between both acoustic approaches and the measurement. The investigated operating point is characterized by the low tip-speed ratio of $\lambda=0.4$ and $R e_{c}=28000$. This flow configuration causes high dynamic stall at the blades. The main sources of the $\mathrm{H}$-Darrieus sound pressure field were identified as the already mentioned separationstall noise and the blade vortex interaction. Future investigations will focus on the acoustic at the optimum tip-speed ratio. Using these CFD and CAA tools, aerodynamic and aeroacoustic optimization can be accomplished with regard to the design of vertical axis wind turbines.

\section{ACKNOWLEDGEMENTS}

The authors would like to thank the reviewers for their informative and detailed comments on the paper, which were very helpful to improve this work.

This work is supported by the Bavarian research project ElHome-Center [27], which is funded by the Bavarian government. 


\section{REFERENCES}

[1] J.F. Manwell, Wind Energy Explained - Theory, Design and Application $2^{\text {nd }}$ Edition, John Wiley \& Sons Ltd. , 2009

[2] I. Paraschivoiu, Wind Turbine design - With Emphasis on Darrieus Concept, Presses Internationales Polytechnique, 2002

[3] D. Milborrow, Vertical axis wind turbines, WindStats Newsletter 8, 1995, pp. 5-8

[4] T. F. Brooks, D. S. Pope, A. Marcolini, Airfoil Self-Noise and Prediction, NASA Reference Publication 1218, 1989

[5] S. Wagner, R. Bareiss and G. Guidati, Wind turbine noise, Springer, 1996

[6] M. H. Mohamed, Performance investigation of H-rotor Darrieus Turbine with new airfoil shapes, Energy, 47, 2012, 522-530

[7] C. J. Ferreira, The near wake of the VAWT $-2 D$ and $3 D$ views of the VAWT aerodynamics, $\mathrm{PhD}$ thesis, TU Delft, 2009

[8] S. Mertens, Wind Energy in the Built Environment $\mathrm{PhD}$ thesis, TU Delft, 2006

[9] M. Marnett, Multiobjective Numerical Design of Vertical Axis Wind Turbine Components $\mathrm{PhD}$ thesis, RWTH Aachen, 2012

[10] A. Iida, A. Mizuno and K. Fukudome, Numerical Simulation of Aerodynamic Noise Radiated from Vertical Axis Wind Turbines, Technical Report, Kogakuin University Department of Mechanical Engineering, 2004

[11] C. Pearson and W. Graham, Investigation of the noise sources on a vertical axis wind turbine using an acoustic array, 19 $19^{\text {th }}$ AIAA/CEAS Aeroacoustics Conference, Berlin, 2013

[12] M. H. Mohamed, Aeroacoustics noise evaluation of H-rotor Darrieus wind turbines, Energy, 65, 2014, 596-604

[13] ANSYS, ANSYS, fourteenth ed, <www.ansys.com>

[14] M. Kaltenbacher, M. Escobar, I. Ali and S. Becker, Numerical Simulation of Flow-Induced Noise Using LES/SAS and Lighthill's Acoustics Analogy, International Journal for Numerical Methods in Fluids, Vol. 63(9), 2010, pp. $1103-1122$

[15] C. Scheit, B. Karic and S. Becker, Effect of blade wrap angle on efficiency and noise of small radial fan impellers - A computational and experimental study Journal of Sound and Vibration, No. 331, No. 5, 2012, pp. 996-1010

[16] W.A. Timmer, Two-dimensional low-Reynolds number wind tunnel results for airfoil NACA0018, Wind Engineering, Vol. 32, No. 6, 2008, pp. 525-537

[17] F. Menter and Y. Egorov, The Scale-Adaptive Simulation Method for Unsteady Turbulent Flow Predictions. Part 1: Theory and Model Description, Flow Turbulence and Combustion, Vol. 85, No. 1, 2010, pp. 113-138

[18] F. Menter et al, The Scale-Adaptive Simulation Method for Unsteady Turbulent Flow Predictions. Part 2: Application to Complex Flows Flow, Turbulence and Combustion, Vol. 85, No. 1, 2010, pp. 139-165 
[19] R. Courant, K. Friedrichs and H. Lewy, Über die partiellen Differentialgleichungen der mathematischen Physik, Mathematischen Annalen, Vol. 100, No. 1, 1928, pp $32-74$

[20] J.M. Lighthill, On Sound Generated Aerodynamically I. General Theory, Proceedings of the Royal Society, Vol. 211, No. 1107, 1952, pp. 564-587.

[21] J. M. Lighthill, On Sound Generated Aerodynamically II. Turbulence as a Source of Sound, Proceedings of the Royal Society, 1954, pp. 1-32.

[22] N. Curle, The Influence of Solid Boundaries upon Aerodynamic Sound, Proceedings of the Royal Society, Vol. 231, No. A, 1955, pp. 505-514.

[23] J.E. Ffowcs Williams and D. L. Hawkings, Sound Generation by Turbulence and Surfaces in Arbitrary Motion, Philosophical Transactions of the Royal Society, Vol. 264, No. 1151, 1969, pp. 321-342.

[24] K. Ehrenfried, Strömungsakustik (Aeroacoustics), Mensch \& Buch Verlag, 2004, pp. $145-159$

[25] F. Farassat, Introduction to generalized functions with applications in aerodynamics and aeroacoustics, Technical Report, 1996, NASA

[26] P. Di Francescantonio, A New Boundary Integral Formulation for the Prediction of Sound Radiation, Journal Sound and Vibration, Vol.202, No. 4, 1997, pp. 491-509.

[27] ElHome-Center, www.ehome-center.de

[28] F. Menter, Best Practice: Scale-Resolving Simulations in ANSYS CFD, Technical Report, Version 1.0, ANSYS, Inc., 2012 